\section{Eutectic Melts Improve Stability of Electrolytes Used in Dye- Sensitized Solar Cells}

Dye-sensitized solar cells (DSCs) are rapidly emerging as a low-cost alternative to traditional silicon photovoltaics. Based on a photoelectrochemical process that is analogous to photosynthesis, DSCs have intrinsically lower fabrication costs and potentially very high efficiencies. However, one key barrier to their wider commercial adoption and large-scale use is the fact that they incorporate volatile, plasticpermeating solvents. Now a group led by the original inventor of the DSC, M. Grätzel of the Swiss Federal Institute of Technology in Lausanne, and P. Wang of the Chinese Academy of Sciences in Changchun have introduced the use of a eutectic melt to produce solvent-free DSCs with efficiencies that approach the highest reported to date. They reported their findings in the August issue of Nature Materials (DOI: 10.1038/nmat2224; p. 626).

The use of solvents in electrolytes has been understood as an obstacle to largescale adoption of DSCs for some time, and a number of researchers have pursued alternatives such as solvent-free roomtemperature ionic liquids. To date, the highest-efficiency solvent-free DSCs have been based on 1-propyl-3-methylimidazolium iodide (PMII), which has a lower viscosity than other iodide melts, enabling high fill factors and no mass-transport limitations on photocurrent. The Lausanne/ Changchun group observed that three other salts that are solid at room temperature, 1-ethyl-3-methylimidazolium iodide (EMII); 1,3-dimethylimidazolium iodide (DMII); and 1-allyl-3-methylimidazolium iodide (AMII), are more conductive than PMII above their melting points. This inspired the researchers to mix EMII, DMII, and AMII at a molar ratio of 1:1:1, resulting in a eutectic-based melt with melting point below $0^{\circ} \mathrm{C}$ and a conductivity of $1.68 \mathrm{mS} \mathrm{cm}^{-1}$, almost three times higher than that of PMII.

The group then fabricated a DSC device using this eutectic-based melt (with routine additives guanidinium thiocyanate and N-butylbenzoimidazole), as well as

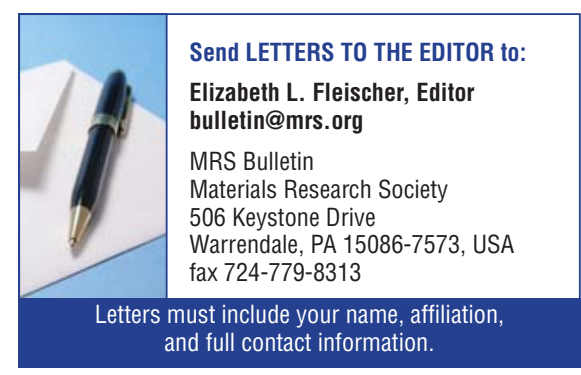

one based on a melt of EMII, DMII, and 1-ethyl-3-methylimidazolium tetracyanoborate (EMITCB). The first device yielded an overall power conversion efficiency in full sunlight of $7.1 \%$, significantly better than corresponding devices based on a PMII electrolyte. The second device displayed overall efficiency of $8.2 \%$, the highest reported to date for a DSC with a solvent-free electrolyte, and retained $93 \%$ of this conversion efficiency after 1000 hours of light soaking with full solar intensity at $60^{\circ} \mathrm{C}$, confirming that it is highly stable.

These results, and particularly the introduction of the use of eutectic melts to produce electrolytes, offer important clues for further improving the stability and efficiency of DSCs. They may also one day be seen as a critical step on the path to large-scale deployment of lowcost photovoltaics.

\section{COLIN MCCORMICK}

\section{First Principles and Finite Element Calculations Combined to Model Charged Dopants in Nanowires}

Semiconducting nanowires (NWs) typically require doping to make them useful in nanoelectronic devices. First principles modeling efforts of carrier transport in NWs (and in carbon nanotubes), however, have heretofore only considered neutral defects because of the computational difficulties related with periodic boundary conditions (PBCs). The long electrostatic screening lengths of charged dopant impurities require huge periodic cells and, therefore, prohibitively expensive calculations. Small periodic cells that incompletely screen out charges lead to divergent Coulomb energies and to physically incorrect interaction between periodic images. Recently, however, R. Rurali from the Universitat Autònoma de Barcelona; T. Markussen of the Technical University of Denmark, A.-P. Jauho of Helsinki University of Technology, Finland, and their colleagues have developed an approach that uses finite element (FE) calculations to correct the potential obtained with density functional theory (DFT) at points far from the charged dopant. The corrected potential is then used to investigate the effect of charged dopant impurities on electron transport.

As reported in the September issue of Nano Letters (DOI: 10.1021/nl801409m; p. 2825), Rurali and co-researchers modeled Si NWs with a diameter of $\sim 2 \mathrm{~nm}$, oriented along the $<100>$ axis, which approximate the thinnest wires grown to date. DFT, as implemented in a previously published software code, was used to calculate the atomic and electronic structures of the NWs. Subsequently, techniques based on recursive Green's functions were used to calculate electron transport, that is, the NW conductance. The researchers simulated $n$-type and $p$-type doping with $\mathrm{B}^{-}$and $\mathrm{P}^{+}$substitutional dopants at the innermost regions of the NWs. Because the electrostatic potential, $V$, converges slowly for single-dopant simulations, the researchers calculated the change in the potential, $\Delta V$, between NWs with and without the substitutional dopant. For supercells composed of five- and nine-unit cells, the researchers found that the DFT potential around the dopant is well converged and does not change significantly with increasing cell size, and that a few nanometers from the dopant, $\Delta V$ from DFT calculations is reproduced remarkably well by inexpensive FE calculations (although poorly in the vicinity of the dopant).

The researchers found that while the $\mathrm{P}^{+}$ dopant qualitatively reproduces the majority-carrier transmission profile of a neutral dopant, the $\mathrm{B}^{-}$dopant can reduce the transmission by up to $50 \%$. For the minority carriers, both dopants constitute an effective barrier in the potential landscape, increasing minority-carrier scattering by a factor of 1000 . The researchers said that these findings "have far-reaching consequences for nanodevices that rely on minority carrier transport, such as nanowire solar cells. On the other hand, a scattering rate three orders of magnitude larger for minority than for majority carriers can be exploited for reducing the off-current in Schottky barriers, thereby improving the performance of metalsemiconductor junctions."

STEVEN TROHALAKI

\section{Revisiting Copper (I) Sulfide for Use in Photovoltaics}

The challenge of the solar energy future is to replace single-crystalline silicon with a low-cost, high-performing alternative. The first generation of alternatives approached the dilemma by using thin films of different semiconductor materials, such as cadmium sulfide (CdS) and copper (I) sulfide $\left(\mathrm{Cu}_{2} \mathrm{~S}\right)$. However, after much investigation into the use of $\mathrm{Cu}_{2} \mathrm{~S} / \mathrm{CdS}$ films, interest in this system declined because of poor long-term performance and low stability. As nanocrystals emerge as a method for constructing solar cells, the use of $\mathrm{Cu}_{2} \mathrm{~S} / \mathrm{CdS}$ is once again a potential photovoltaic material. At the current time there is little published work on the fabrication and photoelectrochemical properties of $\mathrm{Cu}_{2} \mathrm{~S}$ nanocrystals. Y. Wu, C. Wadia, W. Ma, B. Sadtler, and A.P. Alivisatos from the University of California-Berkeley provide insight into a method of manufacturing $\mathrm{Cu}_{2} \mathrm{~S}$-nano- 